\title{
Material structure, properties, and dynamics through scanning transmission electron microscopy
}

\author{
Stephen J. Pennycook ${ }^{1 *}$ D, Changjian $\mathrm{Li}^{1}$, Mengsha $\mathrm{Li}^{1}$, Chunhua Tang ${ }^{1}$, Eiji Okunishi ${ }^{2}$, Maria Varela ${ }^{3}$, \\ Young-Min $\mathrm{Kim}^{4}$ and Jae Hyuck Jang ${ }^{5}$
}

\begin{abstract}
Scanning transmission electron microscopy (STEM) has advanced rapidly in the last decade thanks to the ability to correct the major aberrations of the probe-forming lens. Now, atomic-sized beams are routine, even at accelerating voltages as low as $40 \mathrm{kV}$, allowing knock-on damage to be minimized in beam sensitive materials. The aberration-corrected probes can contain sufficient current for high-quality, simultaneous, imaging and analysis in multiple modes. Atomic positions can be mapped with picometer precision, revealing ferroelectric domain structures, composition can be mapped by energy-dispersive X-ray spectroscopy (EDX) and electron energy loss spectroscopy (EELS), and charge transfer can be tracked unit cell by unit cell using the EELS fine structure. Furthermore, dynamics of point defects can be investigated through rapid acquisition of multiple image scans. Today STEM has become an indispensable tool for analytical science at the atomic level, providing a whole new level of insights into the complex interplays that control material properties.
\end{abstract}

Keywords: Scanning transmission electron microscopy, Electron energy loss spectroscopy, Energy loss near-edge fine structure, Energy-dispersive X-ray spectroscopy, Ferroelectric domain structures, Lead-free piezoelectrics, Point defect dynamics, Nanofabrication

\section{Introduction}

With the successful correction of lens aberrations, the STEM has become the dominant microscopy technique used today in material research, due to the availability of simultaneous, multiple, imaging and spectroscopic modes. While these benefits have long been appreciated in principle (Crewe 1966; Crewe et al. 1970; Rose 1974), before aberration correction, it was difficult to get sufficient current into the probe for good quality images, nor could spectroscopic signals be obtained at atomic resolution. Aberration correction, bringing smaller, brighter probes, has overcome the historic disadvantage of STEM, that of poor signal to noise ratio (Pennycook and Nellist 2011). In this review, we highlight some recent achievements and applications to materials. More

\footnotetext{
* Correspondence: msepsj@nus.edu.sg

${ }^{1}$ Department of Materials Science and Engineering, National University of Singapore, Block EA 07-14, 9 Engineering Drive 1, Singapore 117575, Singapore

Full list of author information is available at the end of the article
}

detailed accounts can be found in a number of recent reviews (Pennycook 2015; Oxley et al. 2016; Varela et al. 2017; Gazquez et al. 2017; Li et al. 2017).

Figure 1 shows the principle of STEM. Like a scanning electron microscope, an incident probe is scanned across a sample, but it is thin enough so that the beam is transmitted, then several signals can be detected simultaneously and used to form images with complementary characteristics. The high-angle annular dark-field (HAADF) detector collects Rutherford scattering from the atomic nuclei, producing an image with strong sensitivity to atomic number $\mathrm{Z}$, often called a Z-contrast image. Light columns such as $\mathrm{O}$ are only weakly visible in the Z-contrast image, but are seen clearly in a simultaneous bright-field (BF) image or annular bright-field (ABF) image. In perovskites and related materials, this allows the octahedral rotations to be determined, which are crucial to understanding their properties. If the bright-field detector is removed, electrons can be passed 


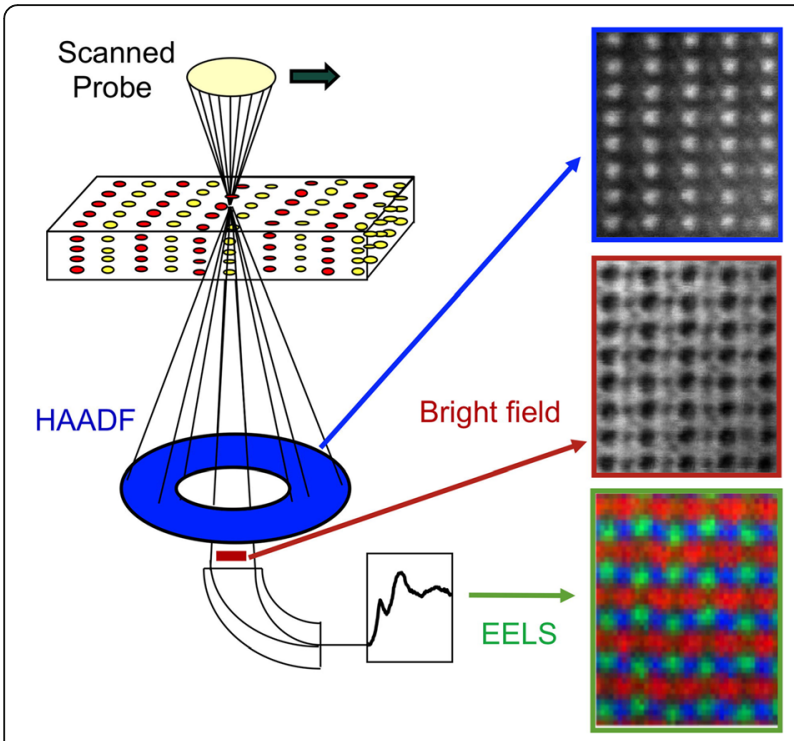

Fig. 1 Schematic showing the multiple imaging and analytical signals available in STEM. Top right is a HAADF image of $\mathrm{BiFeO}_{3}$; the brightest atom columns are Bi and the less bright ones Fe, which are seen displaced slightly to the right due to the ferroelectric polarization. The center image is a bright-field image showing $\mathrm{O}$ columns, below is a color composite EELS image of $\mathrm{LaMnO}_{3}$ in which the $\mathrm{Mn}$ is shown in red, $\mathrm{O}$ in green, showing displacements due to octahedral rotations, and La in blue. Adapted with premission from Borisevich et al. 2010, copyrighted by the American Physical Society, and Varela et al. 2012

through a magnetic sector electron energy loss spectrometer to provide elemental maps and electronic structure information.

\section{Imaging and spectroscopic modes Imaging modes}

The HAADF image is an incoherent image, that is, a direct image, of the columnar scattering power (Pennycook and Boatner 1988; Pennycook and Jesson 1990; Pennycook and Jesson 1991). It is least sensitive to crystal tilts, specimen thickness, and residual aberrations; therefore, it can provide positions of high- $\mathrm{Z}$ columns with high accuracy, as demonstrated in Fig. 2 showing $\mathrm{BiFeO}_{3}$ (BFO) viewed along the pseudocubic [110] direction (denoted $[110]_{\mathrm{pc}}$ ). However, for O columns, which scatter weakly to the HAADF detector, a bright-field image gives more accurate positions, although, being a phase-contrast image, the correct defocus and thickness combination must be determined by image simulation. Combining the positions from both (simultaneously acquired) images allows the octahedral rotations to be accurately measured (Wang et al. 2016; Kim et al. 2017).

The annular bright-field image, a phase-contrast image first proposed by Rose, (Rose 1974) has also become popular in the aberration-corrected era for imaging light atoms (Findlay et al. 2010; Ishikawa
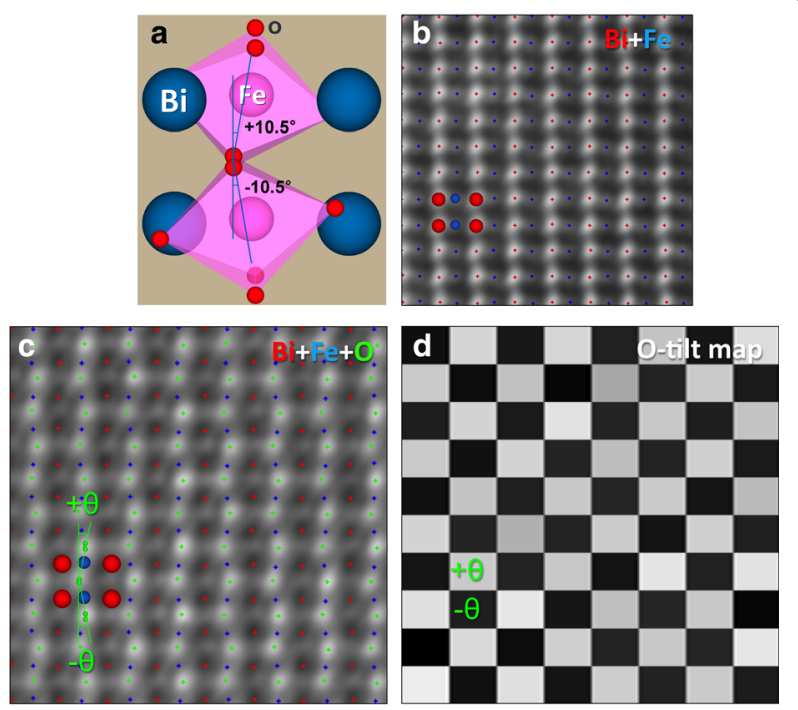

Fig. 2 Measurement of oxygen octahedral tilts in BFO using HAADF and BF STEM images. a Schematic of the BFO structure observed along the $[110]_{p c}$ direction. $\mathbf{b}$, c Simultaneously acquired HAADF and BF STEM images overlaid with peak finding results for the atomic columns. Bi (red) and Fe (blue) atomic columns can be defined from the HAADF STEM image (b) and oxygen columns from the BF STEM image (c). d A checkerboard pattern generated from the measurements of the projected tilt angles $( \pm \theta)$ of oxygen octahedra. Reproduced from Kim et al. 2017. Copyright 2017 with permission from Elsevier

et al. 2011). However, the annular bright-field image may be more sensitive to crystal tilts than a normal bright-field image, as seen from the comparison in Fig. 3 (Kim et al. 2017). Figure 4 shows an example of the application to lead-free piezoelectric materials (Wu et al. 2016). It is known that good properties tend to result from an intimate phase mixture on the nanoscale. Engineering composition to have such phase transitions over the correct temperature range can produce high piezoelectric coefficients; therefore, it is of interest to be able to map local ferroelectric displacements cell by cell to track the polarization rotations. Figure 4 reveals a gradual polarization rotation from a rhombohedral phase (displacement along $<110>$ ) to a tetragonal phase (displacement along < $100>$ ). It is believed that this is indicative of a lowpolarization anisotropy which leads to low-domain wall energy and enhanced piezoelectric coefficient (Zheng et al. 2017). There are many other examples of locating atomic columns to picometer accuracy (Borisevich et al. 2010; Borisevich et al. 2010; Kimoto et al. 2010; Chang et al. 2011; Kim et al. 2012; Yankovich et al. 2014; Tang et al. 2015; Dycus et al. 2015; He et al. 2015; Tang et al. 2016).

Besides accurate location of atomic column positions, much progress has also been made in quantifying crystal 


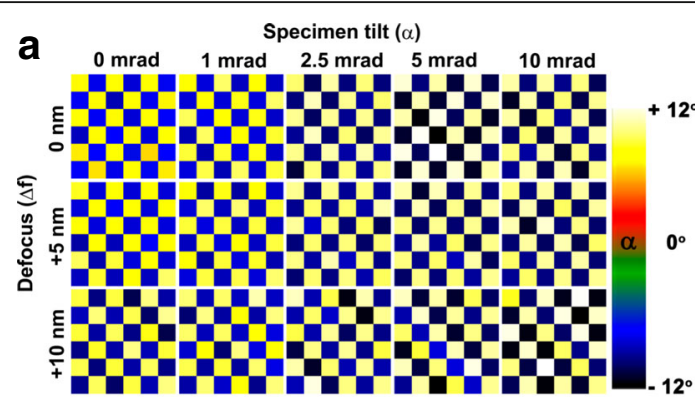

b
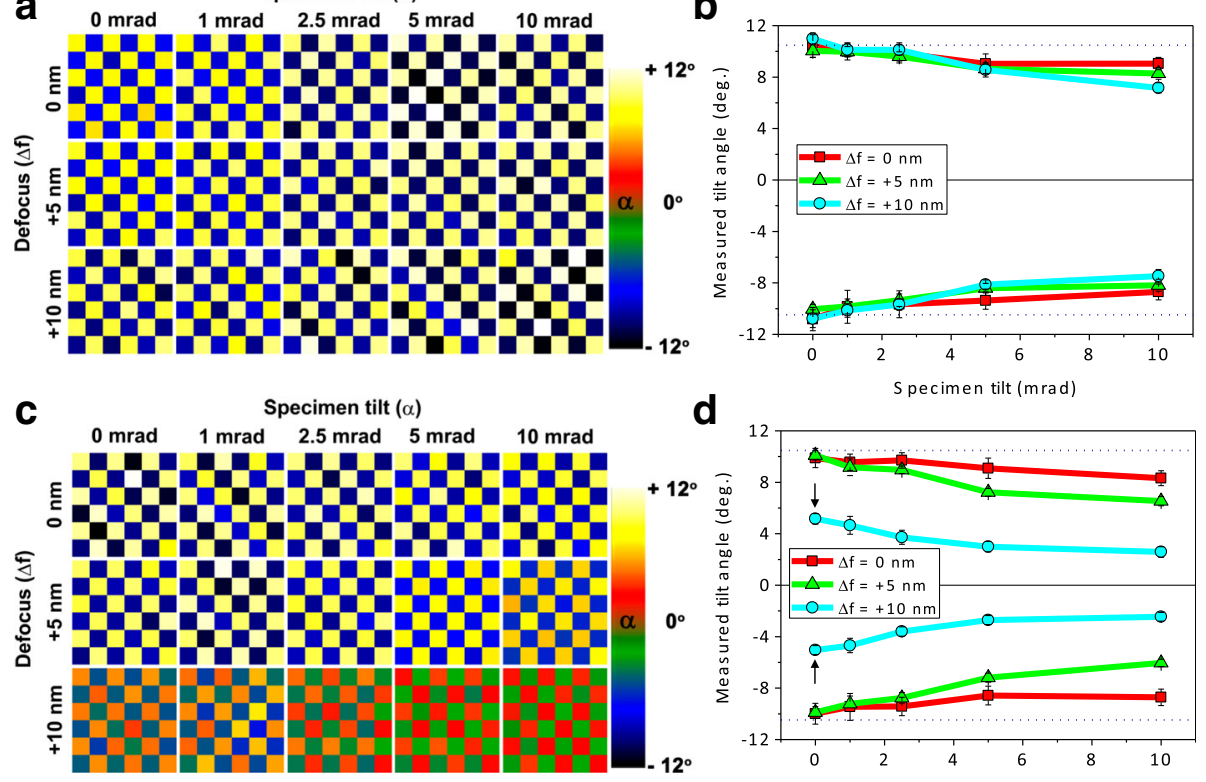

d

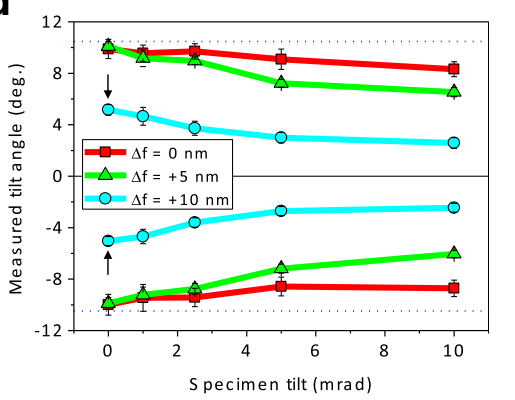

Fig. 3 Effect of specimen tilt in measuring oxygen octahedral tilts in BFO with $10.7 \mathrm{~nm}$ thickness. Oxygen octahedral tilt maps as a function of specimen tilt and defocus calculated from simulated $\mathbf{a} B F$ and $\mathbf{c} A B F$ images, respectively. $\mathbf{b}, \mathbf{d}$ Line profiles of octahedral tilts averaged over vertical rows of the tilt maps shown in $\mathbf{a}$ and $\mathbf{c}$, respectively. The dotted lines in the graphs indicate the tilt value $\left( \pm 10.5^{\circ}\right)$ of bulk BFO for the $[110]_{\mathrm{pc}}$ projection. Simulations for $100 \mathrm{kV}$, spherical aberration coefficient $-0.037 \mathrm{~mm}$, probe semiangle $31 \mathrm{mrad}$, and BF and ABF collection angles 0-1 and 8-20 mrad, respectively. Reproduced from Kim et al. 2017. Copyright 2017 with permission from Elsevier

thickness down to the single-unit cell level, based on column intensity, often referred to as atom counting (Ortalan et al. 2010; Katz-Boon et al. 2013; Van Aert et al. 2013; De Backer et al. 2013; Martinez et al. 2014; Jones et al. 2014; Sang et al. 2014; De Backer et al. 2015). Figure 5 shows an example for a gold crystal (LeBeau et al. 2010). It is even possible to locate an impurity atom in depth with single-unit cell accuracy by accurate fitting of image intensity to simulations (Hwang et al. 2013; Ishikawa et al. 2014). The use of multiple annular detectors provides additional information (Zhang et al. 2015), and recently, it has even been possible to locate vacancies using such quantitative techniques (Kim et al. 2016).

More complex detector geometries are also becoming popular, particularly a segmented detector which allows a differential phase-contrast (DPC) mode (Shibata et al. 2012; Müller et al. 2014; Lazić et al. 2016). Forming the difference signal between opposite segments provides a measure of beam deflection, which can be used to form an image of electric or magnetic fields. Figure 6 shows the imaging of atomic electric fields in $\mathrm{SrTiO}_{3}$, where the fields are seen to point radially outwards from the centers of all the atomic columns, representing the field between the nuclei and the electrons projected along the viewing direction.
A pixelated detector provides another degree of freedom, in that the entire scattered electron distribution can be recorded for each point in the image. Often referred to as $4 \mathrm{D}$ STEM, there are several big advantages although at present, the detector readout severely limits the image speed. One major advantage is that the optimum detection angles can be decided after the image scan, not before as required with scintillator detectors. However, a more fundamental advantage is that not just annuli or segments can be used, but more complex detector patterns. This forms the basis of ptychography, where only those regions that give rise to a particular spatial frequency in the image are selected, as shown in Fig. 7. The method produces better signal to noise ratio and higher contrast than bright-field imaging since the electrons carrying no signal are excluded. Furthermore, no defocus or aberrations are needed to produce the image, so it is ideally complementary to the HAADF image, as shown in Fig. 8.

\section{Analytical modes}

Analytical signals such as energy-dispersive X-ray spectroscopy (EDX) and electron energy loss spectroscopy (EELS) may be orders of magnitude weaker than imaging signals involving scattered electrons, as they typically involve inner shell excitation, which has a 


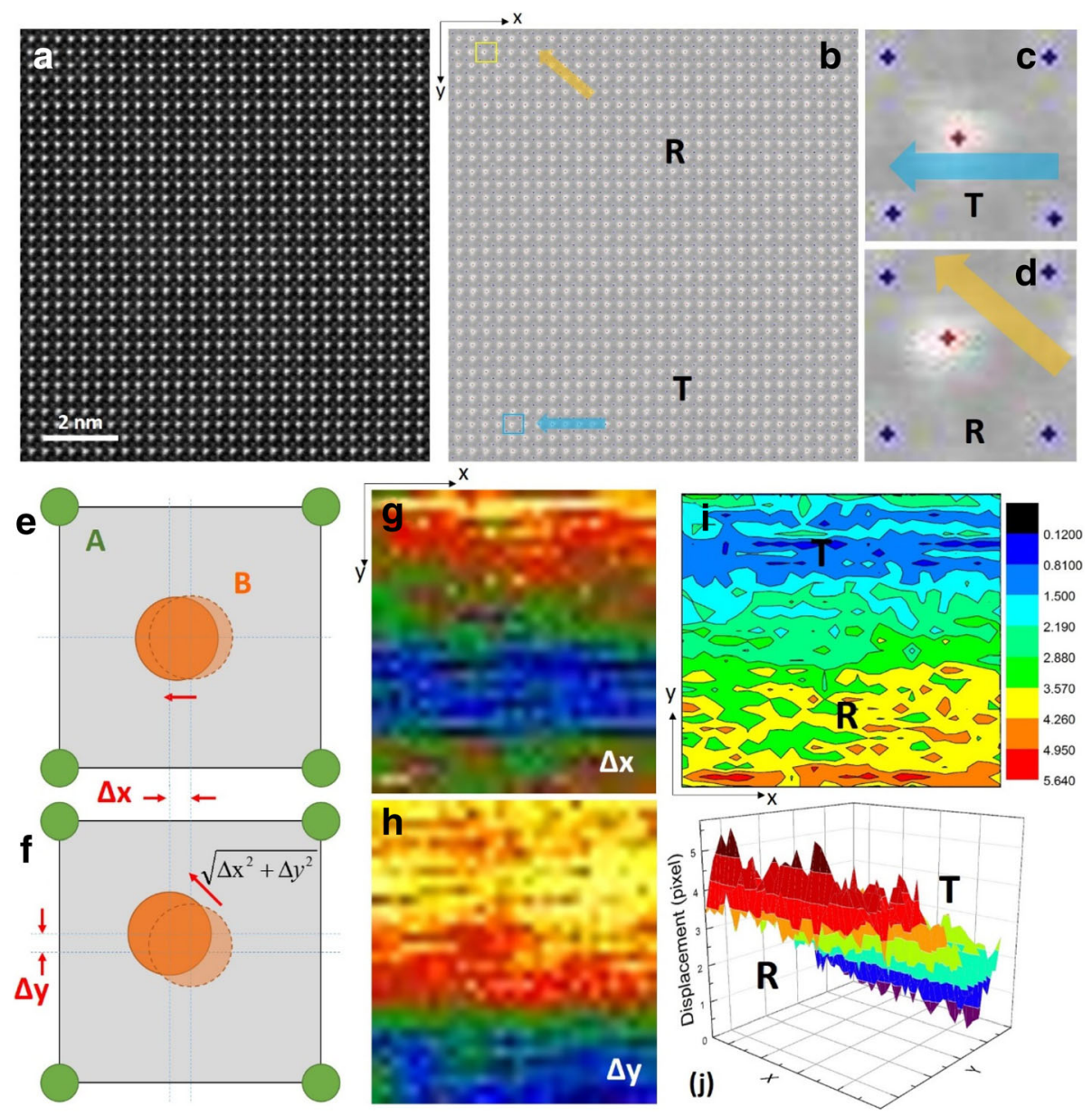

Fig. 4 Local symmetry inside nanodomains of an alkali niobate lead-free ceramic. a STEM HAADF image at a domain boundary; b peak finding on a revealing rhombohedral (R) and tetragonal (T) regions; c enlarged image of the region in a within the blue box, showing $T$ symmetry; $\mathbf{d}$ enlarged image of the region in $\mathbf{b}$ within the yellow box, showing $\mathrm{R}$ symmetry; $\mathbf{e}$, $\mathbf{f}$ schematics showing projected atom displacements for $\mathrm{T}$ and R symmetry; $\mathbf{g}, \mathbf{h}$ displacements along $x$ - and $y$-axes; the region exhibiting displacement along only one axis reflects $T$ symmetry, while the region exhibiting displacement along both $x$ - and $y$-axes reflects $R$ symmetry. $\mathbf{i}, \mathbf{j} 2 \mathrm{D}$ and $3 \mathrm{D}$ images showing displacement along the diagonal direction, reflecting different regions with $\mathrm{T}$ and $\mathrm{R}$ symmetries. Reproduced from Wu et al. 2016. Copyright 2016 American Chemical Society

decreasing cross section with increasing energy loss. Nevertheless, aberration correction allows much larger currents to be focused into atomic-sized probes, as shown in Fig. 9. This fact, combined with major improvements in EDX collection efficiency, have made atomic resolution EDX mapping quite viable, see for example the near-atomically abrupt $\mathrm{SrTiO}_{3} / \mathrm{LaAlO}_{3}$ interface mapped in Fig. 10.

EELS images generally have better statistics because EELS scattering is forward peaked, and for edges not too high in energy, all the inelastically scattered electrons can enter the spectrometer. Hence, it is possible to identify single impurity atoms embedded within a crystal. Figure 11 shows a $0.1 \%$ La-doped film of $\mathrm{CaTiO}_{3}$ grown on $\mathrm{SrTiO}_{3}$ by pulsed laser deposition. Using the La M edge, single La atoms can be located in specific columns of the $\mathrm{CaTiO}_{3}$ crystal.
EELS edges also carry information on electronic structure since the transitions from the core level to the first available empty states depend on atomic valence and environment. This is particularly useful in transition metal oxides since the edge features are linearly related to transition metal valence. Hence, oxidation states can be directly extracted from atomic resolution images by comparison to standard spectra, for example, the O-K edge of a series of $\mathrm{La}_{x} \mathrm{Ca}_{1-x} \mathrm{MnO}_{3}$ compounds with varying $x$, is shown in Fig. 12 (Varela et al. 2009). The first and second peaks can be fitted by Gaussians and parameters such as their ratio or the relative distance between peaks linearly track the Mn oxidation state. This is especially useful for tracking charge transfer across interfaces as shown in Fig. 13, showing EELS data across a $\mathrm{La}_{0.7} \mathrm{Ca}_{0.3} \mathrm{MnO}_{3} / \mathrm{YBa}_{2} \mathrm{Cu}_{3} \mathrm{O}_{7}$. ${ }_{x} / \mathrm{La}_{0.7} \mathrm{Ca}_{0.3} \mathrm{MnO}_{3}(\mathrm{LCMO} / \mathrm{YBCO} / \mathrm{LCMO})$ trilayer (Varela et al. 2017). By quantifying the fine structure, the valence 


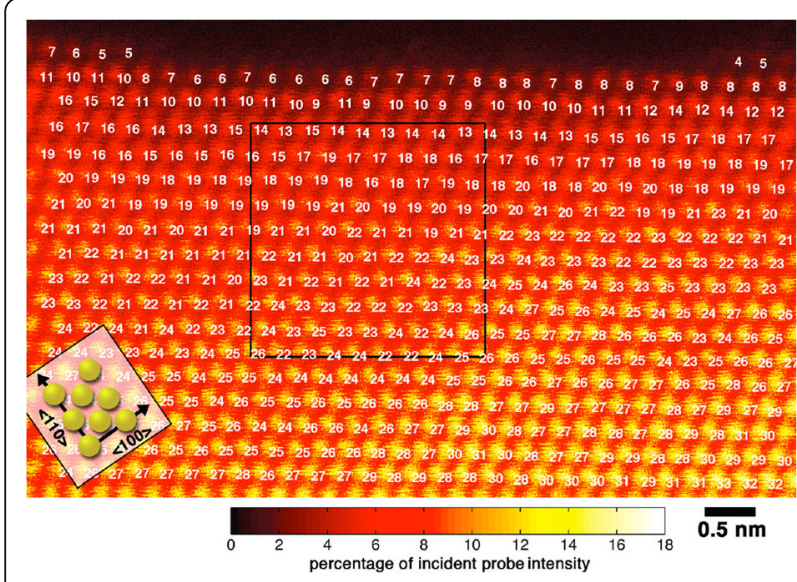

Fig. 5 HAADF-STEM image of a wedge-shaped gold film viewed along $\langle 110\rangle$. The intensity maxima correspond to gold atom columns, and the white labels near the lower right of each atom column indicate the number of atoms contained in that column. The black box outlines the region from which the PACBED pattern shown in Fig. 3 was obtained. The image intensities are shown on an absolute scale relative to the incident beam intensity (see scale bar). Reproduced from LeBeau et al. 2010. Copyright 2010 American Chemical Society

profile can be extracted, and it shows the LCMO layers have a hole concentration slightly higher than bulk while the YBCO layers have a depressed hole concentration, Fig. 14. Hence, electrons have transferred from the LCMO into the $\mathrm{YBCO}$, which is consistent with their respective work functions and explains the depressed critical temperatures in the superconductor (Salafranca et al. 2014). Note also how the normalized pre-peak intensity is higher on the $\mathrm{Cu}-\mathrm{O}$ planes than on the chains, reflecting directly that the holes responsible for superconductivity reside in the planes.

Another recent major advance is in higher energy resolution, achieved through monochromation, which is

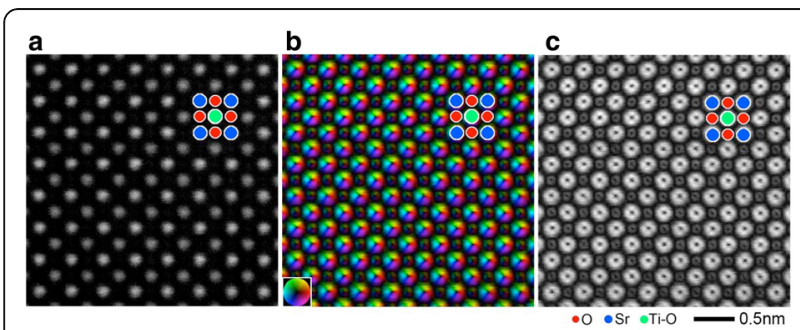

Fig. 6 Simultaneously acquired atomic-resolution STEM images of $\mathrm{SrTiO}_{3}[001]$. a ADF STEM image. b Projected electric field vector color map (left side) and electric field strength map (right side) constructed from the segmented-detector STEM images. The inset color wheel indicates how color and shade denote the electric field orientation and strength in the vector color map. It is seen that both heavy and light element columns are sensitively imaged. Intensity dips are clearly visible at the center of each atomic column position. Images taken with a JEOL ARM-300CF operating at $300 \mathrm{kV}$, adapted from Shibata et al. 2017

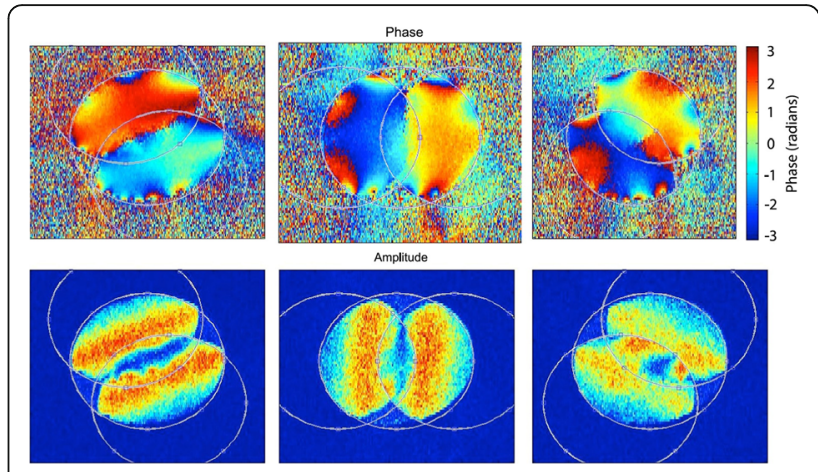

Fig. 7 Ptychographic reconstructions of phase and amplitude components for three spatial frequencies in graphene showing strong contribution from the regions of double overlap. Reproduced from Pennycook et al. 2015. Copyright 2015 with permission from Elsevier

reaching into the meV range (Krivanek et al. 2014), opening the door to phonon spectroscopy (Lagos et al. 2017), and bandgap mapping (Lin et al. 2016). Such energy resolution is comparable to that of a synchrotron, but the microscope provides much better spatial resolution.

However, especially for low losses, the spatial resolution of EELS images may not be as high as for the HAADF or EDX image because electrons only need to pass close enough to the atom to cause an electronic transition, which can occur some distance away, an effect known as delocalization. Egerton (Egerton 2008) has introduced a measure of delocalization as the diameter containing $50 \%$ of the excitations, $d_{50}$. However, it should be noted that this is not the same as image resolution, which is best defined as the full-width-half-maximum of the inelastic image (Oxley et al. 2016). Because of the delocalization effect, EELS images tend to have long tails more resembling a Lorentzian distribution than a Gaussian. The extended tails reduce image contrast more than they reduce resolution. Note also that delocalization is not a simple function of energy loss but depends on the actual electronic transitions. Recently, several examples have been found where low loss images show atomic resolution (Zhou et al. 2012; Zhou et al. 2012; Zhou et al. 2012). Quantum mechanical simulations show that such contrast arises from specific high momentum transfer transitions; hence, there is no violation of the uncertainty principle (Prange et al. 2012; Oxley et al. 2014; Kapetanakis et al. 2015; Kapetanakis et al. 2016).

\section{Dynamics}

The high-energy electron beam can cause ionization of the sample and direct knock-on events (displacement damage). Ionization damage increases as the beam energy is reduced, but knock-on damage decreases, until it 

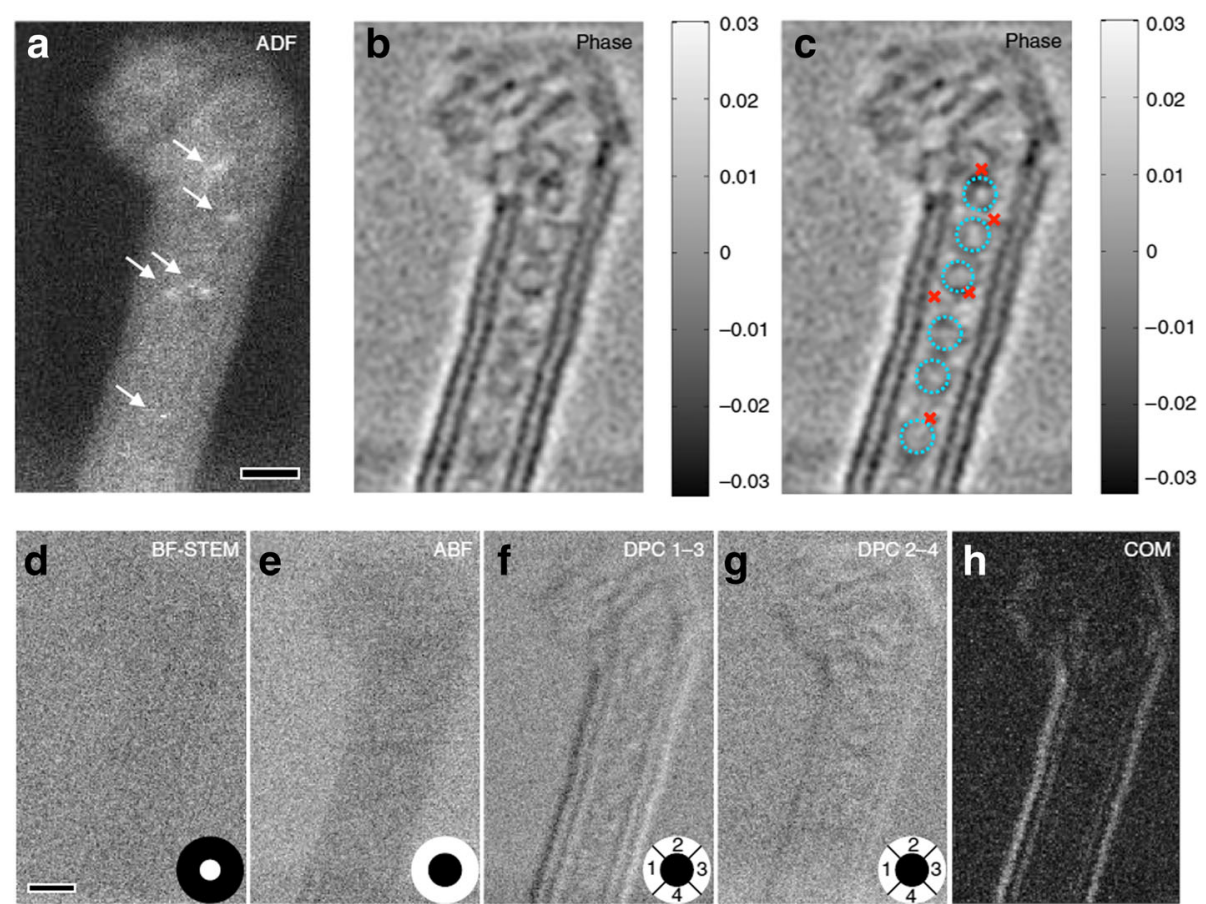

Fig. 8 Simultaneous Z-contrast and phase images of a double-wall carbon nanotube peapod. a Incoherent Z-contrast ADF image clearly shows the locations of the single iodine atoms indicated by the arrows. $\mathbf{b}$ The reconstructed phase image shows the presence of fullerenes inside the nanotube. c Annotated phase image with the fullerenes labeled using dotted circles and iodine atoms labeled using cross marks based on their locations in the ADF image. It is clear that the iodine atoms are located close to but outside the fullerenes. For comparison, conventional phase-contrast images including BF, ABF, DPC, and the DPC using the center of mass approach were synthesized from the data and shown in $\mathbf{d}-\mathbf{h}$, respectively. The detector area of each imaging method is shown in white color in $\mathbf{d}-\mathbf{g}$. The experiment was performed at an electron probe current of $\sim 2.8 \mathrm{pA}$, pixel dwell time of $0.25 \mathrm{~ms}$ and a dose of $\sim 1.310^{4} \mathrm{e} \AA^{-2}$. Scale bar, $1 \mathrm{~nm}$; the gray scale of the phase in $\mathbf{b}$ is in units of radians reproduced from Rutte et al. 2016

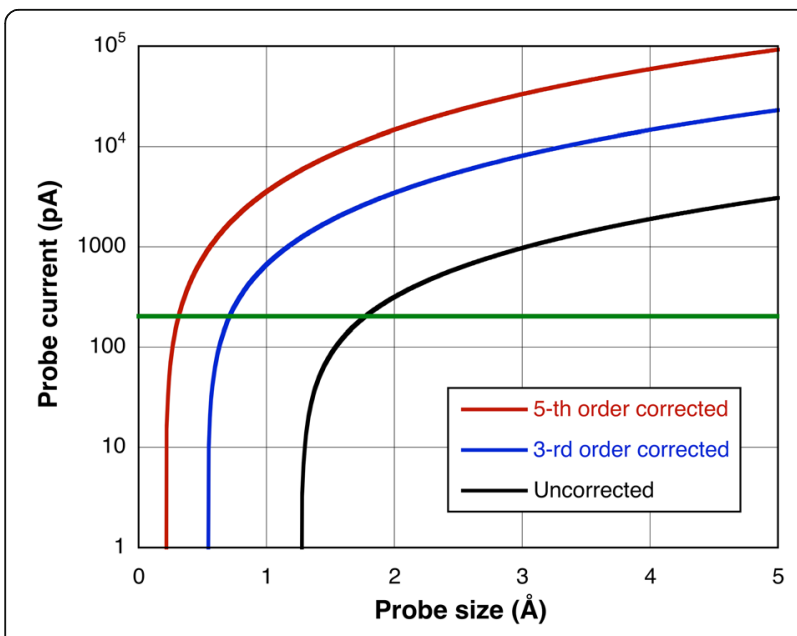

Fig. 9 Variation of probe current with probe size for uncorrected, third- and fifth-order-corrected $300 \mathrm{kV}$ microscopes assuming a source brightness of $3 \times 10^{9} \mathrm{~A} \mathrm{sr}^{-1} \mathrm{~cm}^{-2}$. Below the green line, the probe is predominantly coherent whereas above it is predominantly incoherent. Adapted from Pennycook 2016, with permission from Springer Nature. Copyright 2016

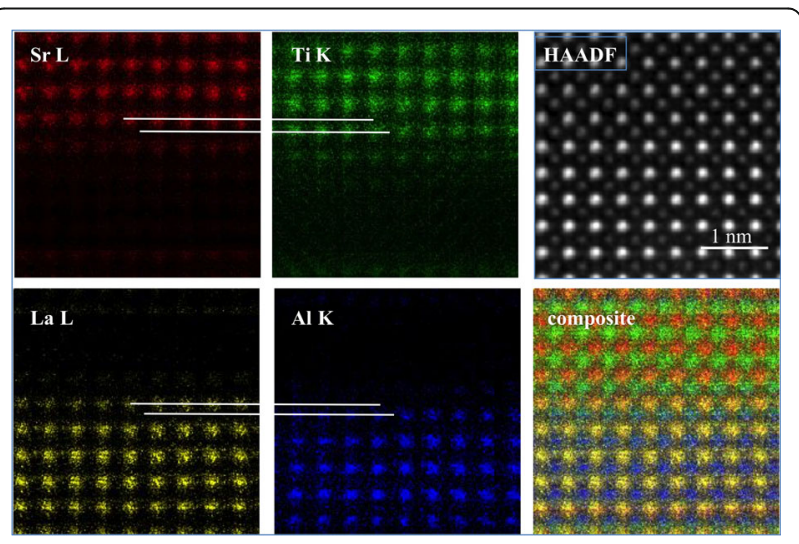

Fig. 10 EDX images of a near-atomically abrupt $\mathrm{SrTiO}_{3} / \mathrm{LaAlO}_{3}$ interface showing atomic columns identified as labeled, with $10 \mathrm{~min}$ total acquisition using an Oxford X-Max 100TLE detector on a JEOL ARM200 equipped with ASCOR aberration corrector operated at $200 \mathrm{kV}$. The composite image includes all edges except O, top right is the HAADF image. Data courtesy Mengsha Li 

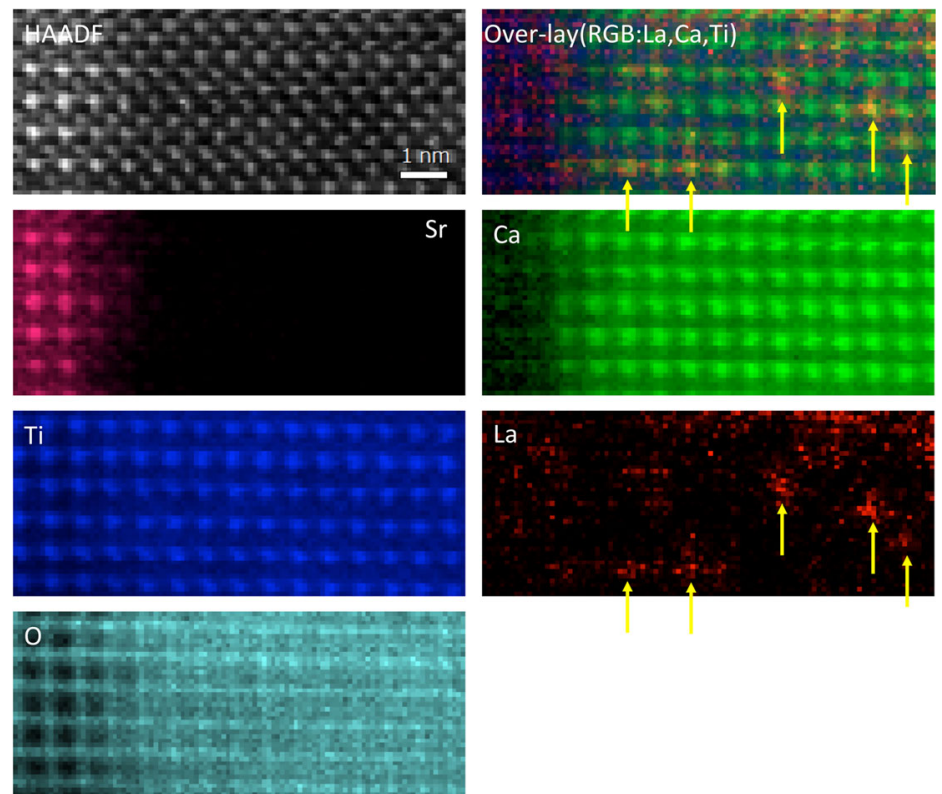

Fig. $11 \mathrm{HAADF}$ image of $\mathrm{La}$-doped $\mathrm{CaTiO}_{3}$ grown on a $\mathrm{SrTiO}_{3}$ substrate with $\mathrm{EELS}$ images resolving the $\mathrm{Ca}$, $\mathrm{Ti}$, and $\mathrm{O}$ columns, and also revealing individual La atoms. The composite color image shows single La atoms in their respective columns. Data obtained with a Gatan Quantum ER on a JEOL ARM 200F equipped with ASCOR aberration corrector operated at $200 \mathrm{kV}$, recorded and processed by E. Okunishi

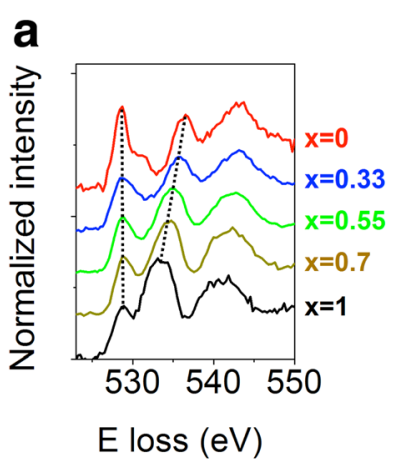

b

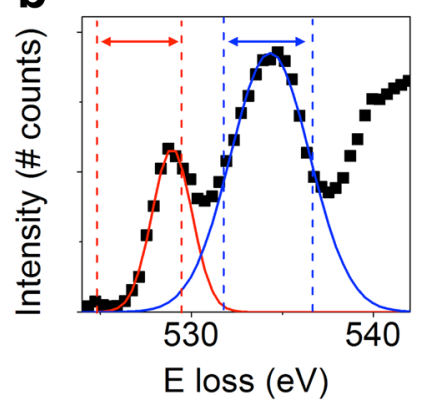

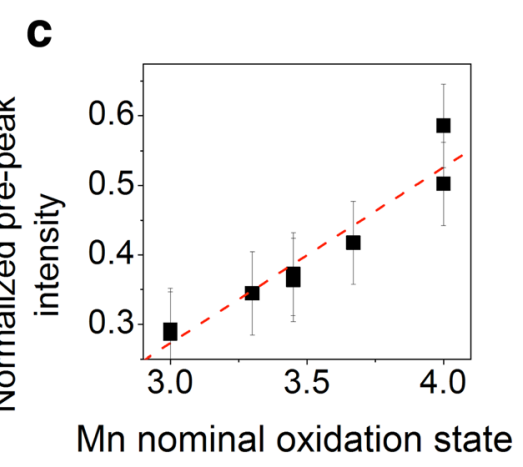

d

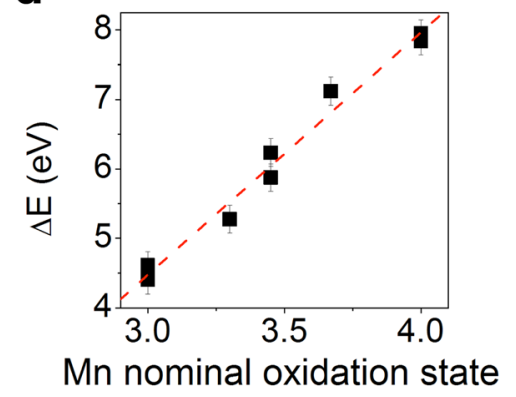

Fig. 12 a O K edges for a series of $\mathrm{La}_{x} \mathrm{Ca}_{1-x} \mathrm{MnO}_{3}$ compounds with $x=1,0.7,0.55,0.33$, and $x=0$ from bottom to top. The energy scale has been shifted, so the pre-peaks are aligned, and the intensity normalized. The spectra have been displaced vertically for clarity. b O K EEL spectrum showing the Gaussian curves used to extract peak intensity and position (pre-peak in red and main peak in blue). c Normalized pre-peak intensity versus nominal oxidation state for the series of LCMO samples. The dashed line is a linear fit to the data. $\mathbf{d}$ Energy separation (calculated as the difference between positions of the second peak and the pre-peak) as a function of the $\mathrm{Mn}$ nominal oxidation state for the sample set of samples. Reprinted with permission from Varela et al. 2009. Copyright 2009 by the American Physical Society 

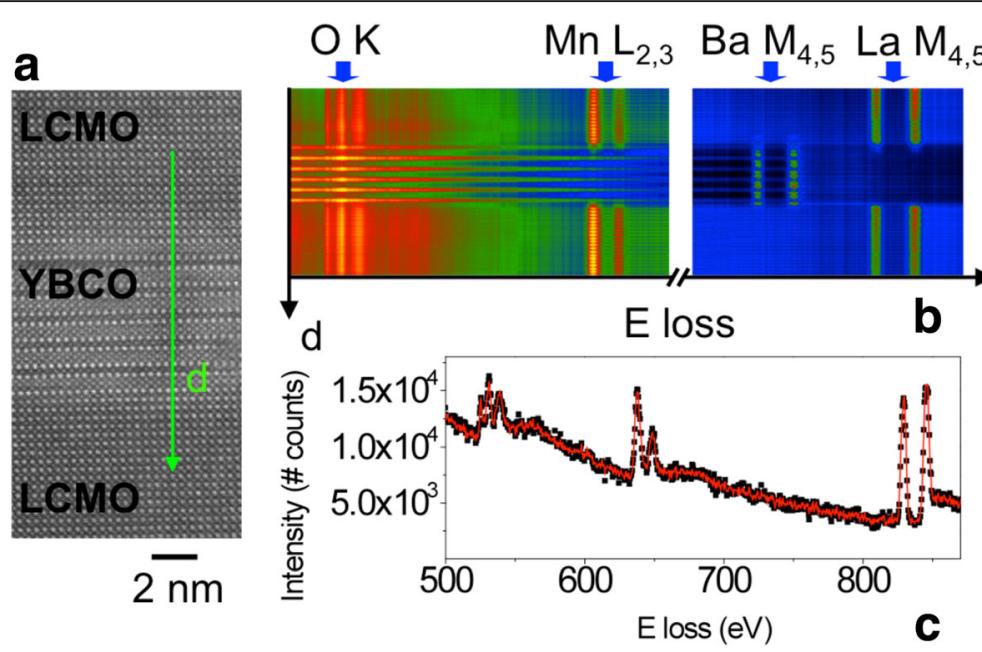

Fig. 13 High-resolution Z-contrast image of a LCMO/YBCO/LCMO trilayer, obtained at $100 \mathrm{kV}$. b EELS linescan acquired along the direction marked with an arrow in (a). Principal component analysis (PCA) has been used to remove random noise. c Sample spectrum extracted from the linescan in (b), acquisition time is $2 \mathrm{~s}$ per spectrum. The data points are a raw spectrum while the red line is the same dataset after PCA. Reproduced from Varela et al. 2012. Copyright 2012 Oxford University Press

disappears entirely below a certain threshold. Historically such processes have been viewed as undesirable damage events, but now that atomic resolution is possible at lower accelerating voltages, there have been many reports of watching atoms move under the beam, allowing insights into atomic motion and the energy landscape of small particles (Kurasch et al. 2012; Komsa et al. 2012; Komsa et al. 2013; Lin et al. 2014; Yang et al. 2014; Guo et al. 2014; Lehtinen et al. 2015; Jesse et al. 2015). Figure 15 shows a time-sequence of images of a
$\mathrm{Si}_{6}$ cluster images at $60 \mathrm{keV}$ beam energy. This is too low to knock atoms out of the cluster, but is sufficient to induce structural changes--one atom is repeatedly seen jumping from the left to right. Such studies reveal metastable configurations that would not be seen by simply heating the material.

Another interesting insight into the dynamics of vacancies is revealed in Fig. 16, which shows beaminduced oxygen vacancy ordering in a $\mathrm{LaCoO}_{3} / \mathrm{SrTiO}_{3}$ (LCO/STO) superlattice (Jang et al. 2017). When O

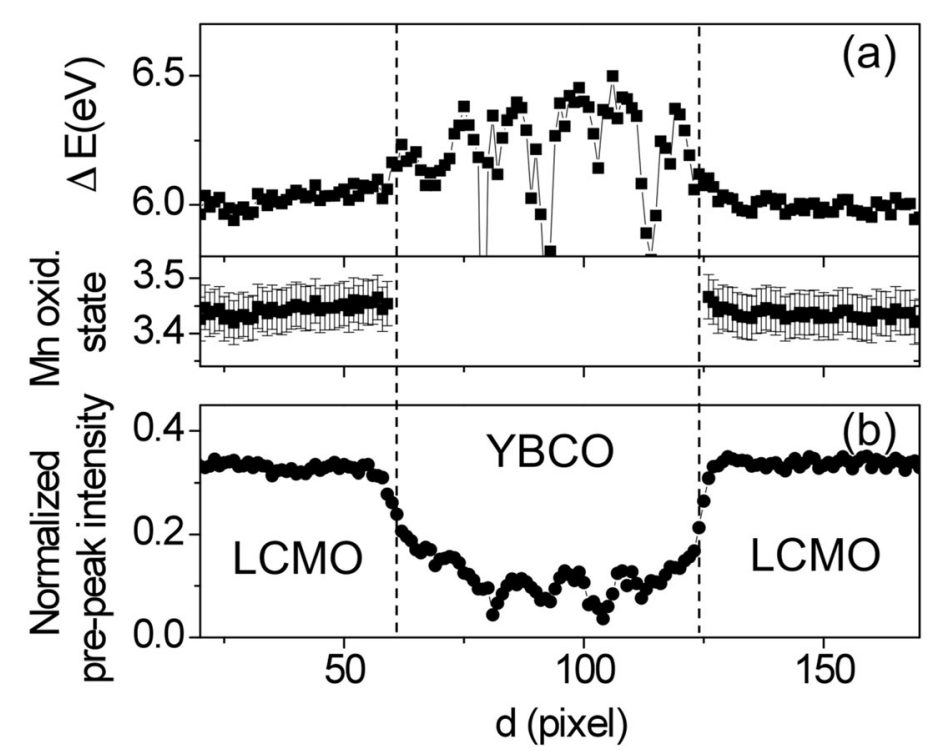

Fig. 14 a Top: For the O K edge, peak separation parameter, measured from the linescan in Fig. 13b. Bottom: Mn oxidation state in the LCMO layers, derived from the data in $\mathbf{a}$. b For the same linescan, O K edge pre-peak normalized integrated intensity. Reproduced from Varela et al. 2012. Copyright 2012 Oxford University Press 

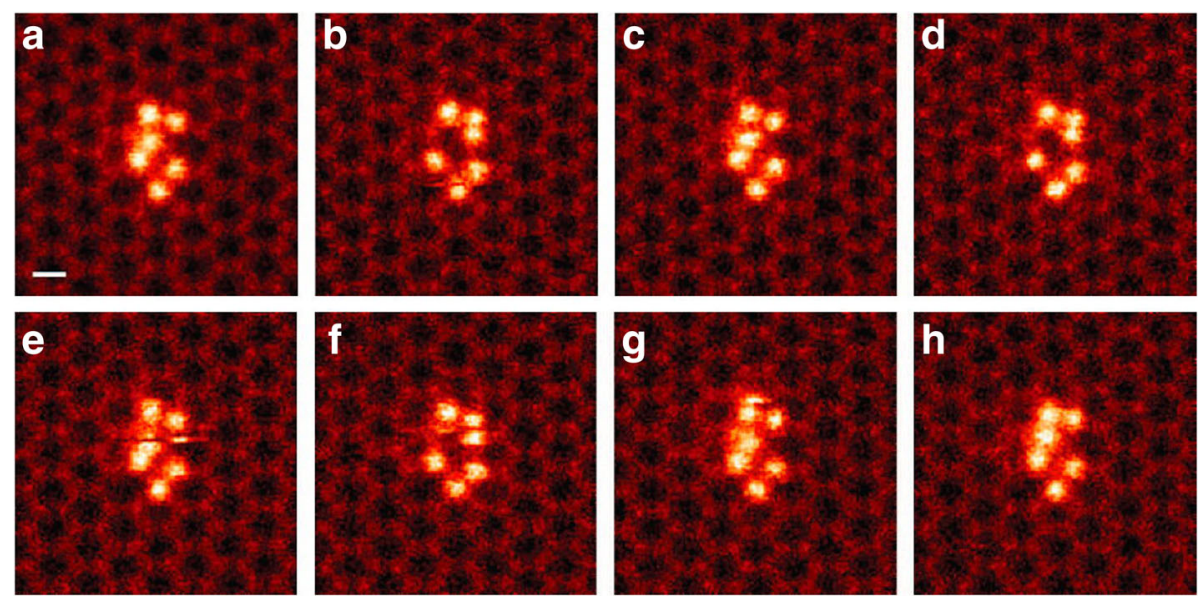

Fig. 15 Sequential STEM Z-contrast images of a $\mathrm{Si}_{6}$ cluster embedded in a graphene pore $(\mathbf{a}-\mathbf{h})$. Scale bar, $0.2 \mathrm{~nm}$. Reproduced from Lee et al. 2013

vacancies order into specific lattice planes, the strain energy is reduced since the lattice spacing of an entire plane can relax. The plane containing the vacancies expands, causing dark contrast in a Z-contrast image (Kim et al. 2012). Tracking planar spacings can therefore reveal this ordering process quantitatively. Since the average spacing in the LCO block in Fig. 16 does not change, the images show ordering of pre-existing vacancies rather than generation of new ones.
An example of the beam-induced diffusion of a heavy Ce atom is shown in Fig. 17. Quantification of image intensities matched to image simulations show that the atom jumps between next-neighbor sites inside the crystal, so the beam-induced motion is seeing the same diffusion processes that are normally induced thermally. Correlated vacancy/Ce atom motion and interstitial knock-out processes have also been seen (Ishikawa et al. 2014). The electron beam can even be used for solid-
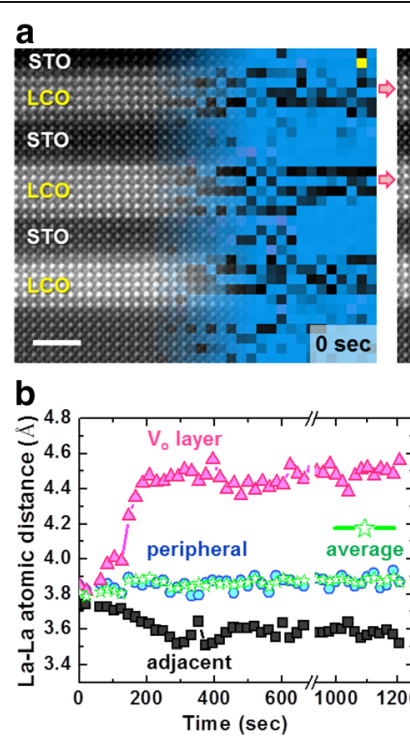

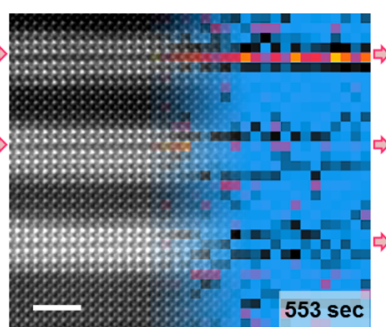

C

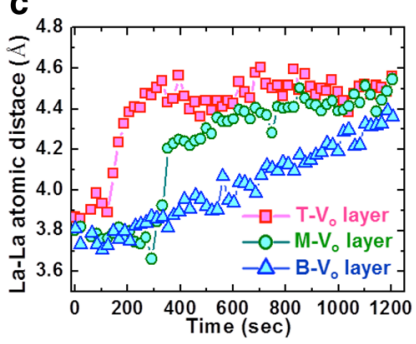

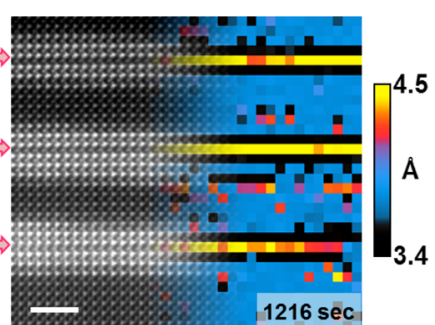

d

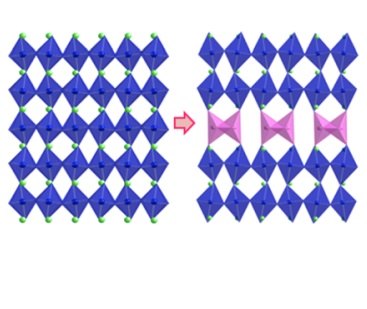

Fig. 16 Beam-induced oxygen vacancy ordering in a $\mathrm{LaCoO}_{3} / \mathrm{SrTiO}_{3}$ superlattice grown on a $\mathrm{SrTiO}_{3}$ substrate viewed along the [100] direction. a Sequential ADF images overlaid with corresponding maps of the out-of-plane interatomic La-La spacings. $\mathbf{b}$ Average spacings in the central $V_{0}$ layer (red curve), the adjacent (black curve), and peripheral (blue curve) layers within the top LCO block as a function of time; green (star) curve gives the overall average, suggesting that the beam primarily induces redistribution of existing vacancies rather than vacancy injection. c Comparison of the evolution of interatomic spacings in the oxygen-depleted planes of the top (T), middle (M), and bottom (B) $\mathrm{LaCoO}_{3}$ blocks; the differences are attributed to differences in thickness due to the wedge geometry of the sample. $\mathbf{d}$ Atomic model of the ordering transition; La atoms are shown in green, $\mathrm{CoO}_{6}$ octahedra in blue, and $\mathrm{CoO}_{4}$ tetrahedra in purple. Reproduced from Jang et al. 2017. Copyright 2017 American Chemical Society 

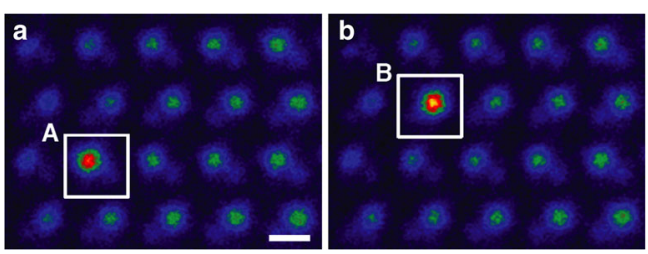

Fig. 17 Atomic-resolution STEM images of Ce hopping from column A to $B$ in AIN. Images are averages over (a) 19 frames before the jump and (b) 19 frames after the jump. Reproduced from Ishikawa et al. 2014. Copyright 2014 American Chemical Society

phase epitaxial crystallization of an amorphous material, as demonstrated by writing the letters ORNL in $\mathrm{SrTiO}_{3}$, see Fig. 18 (Jesse et al. 2015). Such beam-induced nanolithography should be achievable in three dimensions, due to the nanometer depth of focus of the aberrationcorrected probe. Recently, it has been demonstrated that the STEM probe can be used to "push" single impurity atoms through the graphene lattice (Dyck et al. 2017). The potential for single atom fabrication with beams and probes is the subject of a recent issue of MRS Bulletin (Pennycook and Kalinin 2017).

\section{Imaging, analysis, and nanofabrication in 3D}

Ideally, we need atomic scale imaging, analysis, and nanofabrication not just in a two-dimensional projection but in three dimensions. In recent years, much progress has been made in tomography, combining views in multiple directions to reconstruct 3D structure (Goris et al. 2013; Bals et al. 2014; Goris et al. 2015; Miao et al. 2016; Bals et al. 2016). Figure 19 shows an example of the atomic scale reconstruction of an FePt nanoparticle revealing the presence of different ordered structures and different degrees of ordering (Yang et al. 2017). Grain boundaries and even point defects could be detected. Notable progress has also been made in EELS and EDX tomography, although atomic resolution remains far off due to the lower signal levels (Jarausch et al. 2009; Yedra et al. 2012; Haberfehlner et al. 2014; Collins and Midgley 2017).

The example shown in Fig. 19 required many hours of data collection, which many samples could not withstand. An alternative method is optical sectioning. Aberration correctors achieve higher resolution through higher probe convergence angles. Lateral resolution increases linearly with increasing probe angle; however, depth resolution increases quadratically. So, with the latest generation of aberration correctors, the depth of focus has reduced to the nanometer scale, and the image comes from a thin section of the sample. Changing the focus gives a series of images at different depths which could also be reconstructed into a 3D image without the need to tilt the sample. At present, such optical sectioning has not achieved atomic resolution, but may with future generations of aberration corrector (Pennycook and Kalinin 2014; Pennycook 2015; Ishikawa et al. 2016). Figure 20 shows a simulated focal series of $\mathrm{Ce}$ atoms substituted in AlN for probe-forming angles of 30, 60, and $100 \mathrm{mrad}$ (Ishikawa et al. 2015). For the larger probe angles, the two dopant atoms can be easily located at 2and $8-\mathrm{nm}$ depth. Although these simulations assume aberration-free conditions, simulations including chromatic aberration and the effects of electron shot noise

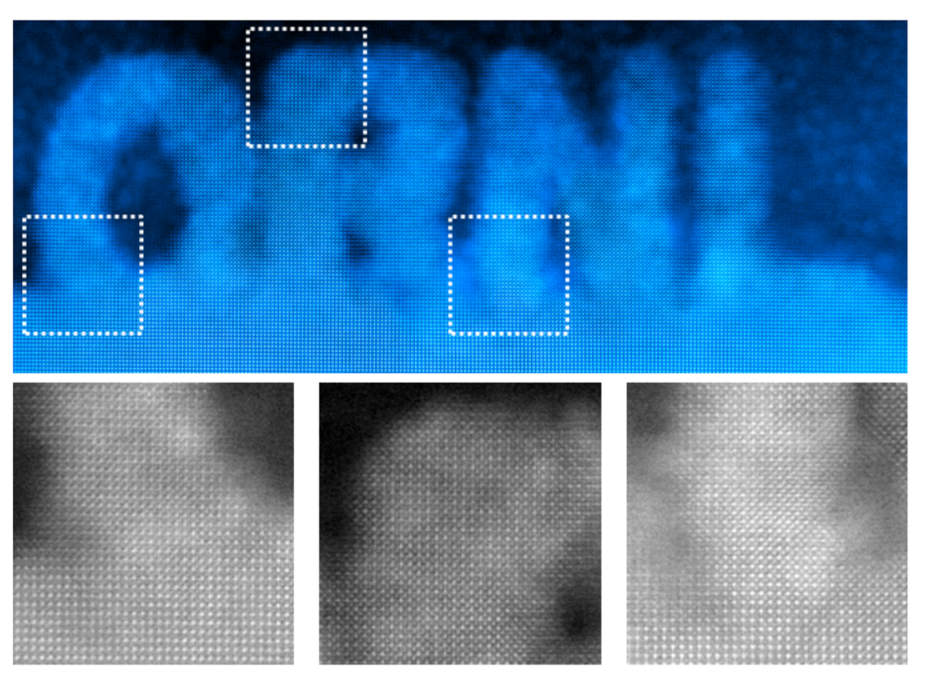

Fig. 18 An example of crystalline oxide sculpting using an arbitrary graphical pattern (in this case, text "ORNL"): Fourier-filtered HAADF image of the (a) complete structure and ( $\mathbf{b}-\mathbf{d})$ magnified raw images of the regions at the top and at the base of the patterned letters. Note the same crystallographic orientation in $\mathbf{b}, \mathbf{c}$, and $\mathbf{d}$, highlighting the epitaxial character of the growth. The widths of image $\mathbf{b}, \mathbf{c}$, and $\mathbf{d}$ are $12 \mathrm{~nm}$. Reproduced from Jesse et al. 2015 


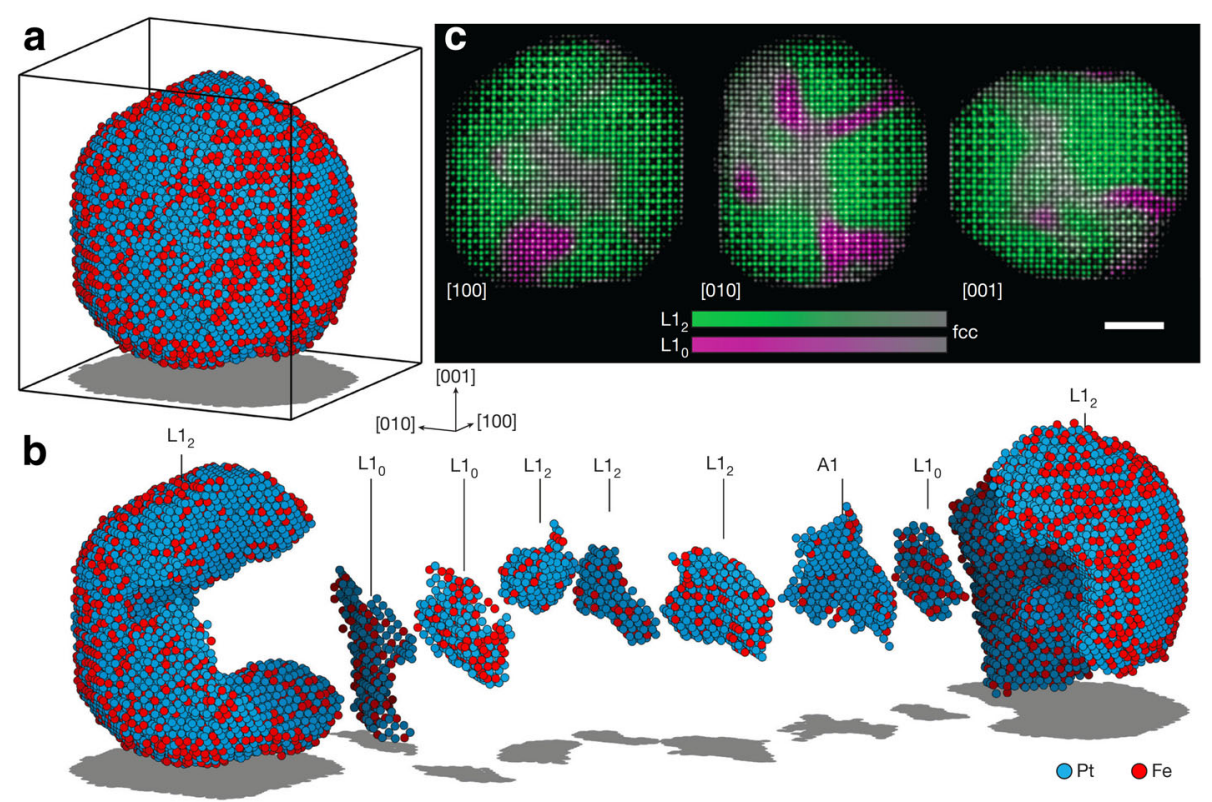

Fig. 19 3D determination of atomic coordinates, chemical species, and grain structure of an FePt nanoparticle. a Overview of the 3D positions of individual atomic species with Fe atoms in red and Pt atoms in blue. b The nanoparticle consists of two large L12 grains, three small L12 grains, three small L10 grains, and a Pt-rich A1 grain. c Multislice image. Reproduced from Yang et al. 2017, by permission from Springer Nature. Copyright 2017

suggest this approach should work under realistic conditions (Ishikawa et al. 2016). Optical sectioning can also be applied to analytical signals with nanoscale resolution, avoiding the need for specimen tilting (Pennycook et al. 2017). Alternatively, it may prove better to combine depth sectioning with discrete tomography (Alania et al. 2017).

\section{Conclusions}

STEM has developed dramatically in recent years, thanks to the development of aberration correctors which have allowed the advantages of multiple, simultaneous imaging and spectroscopic modes to be exploited with high sensitivity and precision. There are advantages also for in situ and operandi studies since STEM allows good control of dose rate and illumination

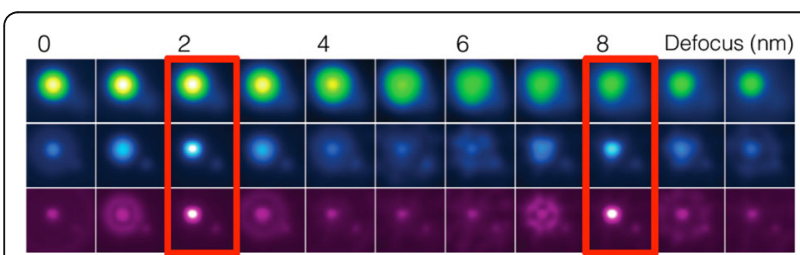

Fig. 20 Simulated focal series of images of Ce atoms substituted in AIN for probe-forming angles of 30 (upper), 60 (center), and 100 (lower) mrad at $300 \mathrm{kV}$ accelerating voltage, assuming aberrationfree conditions. Ce atom locations are indicated by red rectangles. Reproduced from Ishikawa et al. 2015. Copyright 2014, with permission from Elsevier area (Chang et al. 2011; Jungjohann et al. 2012; Mehdi et al. 2015; Wang et al. 2016). There are also major developments in mathematical image reconstruction techniques, learning from other fields such as computer vision, which are pushing towards lower dose imaging (Stevens et al. 2014; Meyer et al. 2014; Kovarik et al. 2016; Voyles 2017). It is certainly an exciting and rewarding time to be exploring the atomic world.

\section{Acknowledgements}

All authors are grateful to their collaborators on the work cited here. SJP is grateful to the National University of Singapore for funding. CJL is supported by a Lee Kuan Yew Fellowship. Research at Universidad Complutense is sponsored by the European Research Council PoC-2016 POLAR-EM and Spanish MINECO-FEDER MAT2015-66888-C3-3-R. YMK was supported by the Institute for Basic Science (IBS-R011-D1) and Creative Materials Discovery Program through the NRF (National Research Foundation of Korea) grant (NRF-2015M3D1A1070672). J.H. Jang is supported by the Korea Basic Science Institute grant (T37210).

\section{Funding}

SJP is grateful to the National University of Singapore for funding. CJL is supported by a Lee Kuan Yew Fellowship. Research at Universidad Complutense is sponsored by the European Research Council PoC-2016 POLAR-EM and Spanish MINECO-FEDER MAT2015-66888-C3-3-R. YMK was supported by the Institute for Basic Science (IBS-R011-D1) and Creative Materials Discovery Program through the NRF (National Research Foundation of Korea) grant (NRF-2015M3D1A1070672) in Korea. J.H. Jang is supported by the Korea Basic Science Institute grant (T37210).

Availability of data and materials Not applicable

\section{Authors' contributions}

All authors contributed to the results reviewed, and all authors have approved the manuscript for submission. 


\section{Competing interests}

The authors declare that they have no competing interests.

\section{Publisher's Note}

Springer Nature remains neutral with regard to jurisdictional claims in published maps and institutional affiliations.

\section{Author details}

${ }^{1}$ Department of Materials Science and Engineering, National University of Singapore, Block EA 07-14, 9 Engineering Drive 1, Singapore 117575 , Singapore. ${ }^{2}$ EM Business Unit, JEOL Ltd., Tokyo 196-8558, Japan. ${ }^{3} D p t$. Física de Materiales, Instituto de Magnetismo Aplicado \& Instituto Pluridisciplinar, Universidad Complutense de Madrid, 28040 Madrid, Spain. ${ }^{4}$ Department of Energy Science, Sungkyunkwan University (SKKU), Suwon 16419, Republic of Korea. ${ }^{5}$ Electron Microscopy Research Center, Korea Basic Science Institute, Daejeon 34133, South Korea.

\section{Received: 14 December 2017 Accepted: 14 March 2018}

\section{Published online: 11 April 2018}

\section{References}

Alania M, Altantzis T, De Backer A, et al. Depth sectioning combined with atomcounting in HAADF STEM to retrieve the 3D atomic structure. Ultramicroscopy. 2017:177:36-42. https://doi.org/10.1016/j.ultramic.2016.11.002.

Bals S, Goris B, Altantzis T, et al. Seeing and measuring in 3D with electrons. Comptes Rendus Physique. 2014;15:140-50. https://doi.org/10.1016/j.crhy. 2013.09.015.

Bals S, Goris B, De Backer A, et al. Atomic resolution electron tomography. MRS Bull. 2016;41:525-30. https://doi.org/10.1557/mrs.2016.138.

Borisevich A, Ovchinnikov OS, Chang HJ, et al. Mapping octahedral tilts and polarization across a domain wall in $\mathrm{BiFeO}_{3}$ from Z-contrast scanning transmission electron microscopy image atomic column shape analysis. ACS Nano. 2010:4:6071-9. https://doi.org/10.1021/nn1011539.

Borisevich AY, Chang HJ, Huijben M, et al. Suppression of octahedral tilts and associated changes in electronic properties at epitaxial oxide heterostructure interfaces. Phys Rev Lett. 2010;105:087204. https://doi.org/10.1103/ PhysRevLett.105.087204

Chang H, Kalinin SV, Yang S, et al. Watching domains grow: in-situ studies of polarization switching by combined scanning probe and scanning transmission electron microscopy. J Appl Phys. 2011;110:052014-7. https:// doi.org/10.1063/1.3623779.

Chang HJ, Chang HJ, Kalinin SV, et al. Atomically resolved mapping of polarization and electric fields across ferroelectric/oxide interfaces by Zcontrast imaging. Adv Mater 2474-2479. 2011; https://doi.org/10.1002/adma. 201004641

Collins SM, Midgley PA. Progress and opportunities in EELS and EDS tomography. 1-9. 2017; https://doi.org/10.1016/j.ultramic.2017.01.003.

Crewe AV. Scanning electron microscopes - is high resolution possible? Science. 1966;154:729-38.

Crewe AV, Wall J, Langmore J. Visibility of single atoms. Science. 1970;168:1338-40.

De Backer A, De Wael A, Gonnissen J, Van Aert S. Optimal experimental design for nano-particle atom-counting from high-resolution STEM images. Ultramicroscopy. 2015;151:46-55. https://doi.org/10.1016/j.ultramic.2014.10.015.

De Backer A, Martinez GT, Rosenauer A, Van Aert S. Atom counting in HAADF STEM using a statistical model-based approach: methodology, possibilities, and inherent limitations. Ultramicroscopy. 2013;134:23-33. https://doi.org/10. 1016/j.ultramic.2013.05.003.

Dyck O, Kim S, Kalinin SV, Jesse S. Placing single atoms in graphene with a scanning transmission electron microscope. Appl Phys Lett. 2017;111: 113104-6. https://doi.org/10.1063/1.4998599.

Dycus JH, Harris JS, Sang $X$, et al. Accurate nanoscale crystallography in realspace using scanning transmission electron microscopy. Microsc Anal. 2015; 21:946-52. https://doi.org/10.1017/S1431927615013732.

Egerton RF. Electron energy-loss spectroscopy in the TEM. Rep Prog Phys. 2008; 72:016502x. https://doi.org/10.1088/0034-4885/72/1/016502.

Findlay SD, Shibata N, Sawada H, et al. Dynamics of annular bright field imaging in scanning transmission electron microscopy. Ultramicroscopy. 2010;110: 903-23. https://doi.org/10.1016/j.ultramic.2010.04.004

Gazquez J, Sanchez-Santolino G, Biškup N, et al. Materials science in semiconductor processing. Mater Sci Semicond Process. 2017;65:49-63. https://doi.org/10.1016/j.mssp.2016.06.005.
Goris B, De Backer A, Van Aert S, et al. Three-dimensional elemental mapping at the atomic scale in bimetallic nanocrystals. Nano Lett. 2013;13:4236-41. https://doi.org/10.1021/nl401945b.

Goris B, De Beenhouwer J, De Backer A, et al. Measuring lattice strain in three dimensions through electron microscopy. Nano Lett. 2015;15:6996-7001. https://doi.org/10.1021/acs.nanolett.5b03008.

Guo J, Lee J, Contescu Cl, et al. Crown ethers in graphene. Nat Commun. 2014;5: 1-6. https://doi.org/10.1038/ncomms6389.

Haberfehlner G, Orthacker A, Albu M, et al. Nanoscale voxel spectroscopy by simultaneous EELS and EDS tomography. Nano. 2014;6:14563-9. https://doi. org/10.1039/C4NR04553J.

He $\mathrm{Q}$, Ishikawa $\mathrm{R}$, Lupini $\mathrm{AR}$, et al. Towards 3D mapping of $\mathrm{BO}_{6}$ octahedron rotations at perovskite heterointerfaces, unit cell by unit cell. ACS Nano. 2015; 9:8412-9. https://doi.org/10.1021/acsnano.5b03232.

Hwang J, Zhang JY, D'Alfonso AJ, et al. Three-dimensional imaging of individual dopant atoms in $\mathrm{SrTiO}_{3}$. Phys Rev Lett. 2013;111:266101. https://doi.org/10. 1103/PhysRevLett.111.266101.

Ishikawa R, Lupini AR, Findlay SD, et al (2014) Three-dimensional location of a single dopant with atomic precision by aberration-corrected scanning transmission electron microscopy. Nano Lett 1903-1908 doi: https://doi.org/ $10.1021 / \mathrm{nl} 500564 \mathrm{~b}$

Ishikawa R, Lupini AR, Hinuma Y, Pennycook SJ. Large-angle illumination STEM: toward three-dimensional atom-by-atom imaging. Ultramicroscopy. 2015;151: 122-9. https://doi.org/10.1016/j.ultramic.2014.11.009.

Ishikawa R, Mishra R, Lupini AR, et al. Direct observation of dopant atom diffusion in a bulk semiconductor crystal enhanced by a large size mismatch. Phys Rev Lett. 2014;113:155501. https://doi.org/10.1103/PhysRevLett.113.155501.

Ishikawa R, Okunishi E, Sawada H, et al. Direct imaging of hydrogen-atom columns in a crystal by annular bright-field electron microscopy. Nat Mater. 2011;10:278-81. https://doi.org/10.1038/nmat2957.

Ishikawa R, Pennycook SJ, Lupini AR, et al. Single atom visibility in STEM optical depth sectioning. Appl Phys Lett. 2016;109:163102. https://doi.org/10.1063/1.4965709.

Jang JH, Kim Y-M, He Q, et al. In situ observation of oxygen vacancy dynamics and ordering in the epitaxial $\mathrm{LaCoO}_{3}$ system. ACS Nano. 2017;11:6942-9. https://doi.org/10.1021/acsnano.7b02188.

Jarausch K, Thomas P, Leonard DN, et al. Four-dimensional STEM-EELS: enabling nano-scale chemical tomography. Ultramicroscopy. 2009;109:326-37. https:// doi.org/10.1016/j.ultramic.2008.12.012.

Jesse $\mathrm{S}, \mathrm{He} \mathrm{Q}$, Lupini AR, et al. Atomic-level sculpting of crystalline oxides: toward bulk nanofabrication with single atomic plane precision. Small. 2015:11:5895900. https://doi.org/10.1002/smll.201502048.

Jones L, MacArthur KE, Fauske VT, et al. Rapid estimation of catalyst nanoparticle morphology and atomic-coordination by high-resolution Z-contrast electron microscopy. Nano Lett. 2014;14:6336-41. https://doi.org/10.1021/nl502762m.

Jungjohann KL, Evans JE, Aguiar JA, et al. Atomic-scale imaging and spectroscopy for in situ liquid scanning transmission electron microscopy. Microsc Anal. 2012;18:621-7. https://doi.org/10.1017/S1431927612000104.

Kapetanakis MD, Oxley MP, Zhou W, et al. Signatures of distinct impurity configurations in atomic-resolution valence electron-energy-loss spectroscopy: application to graphene. Phys Rev B. 2016;94:155449. https:// doi.org/10.1103/PhysRevB.94.155449.

Kapetanakis MD, Zhou W, Oxley MP, et al. Low-loss electron energy loss spectroscopy: an atomic-resolution complement to optical spectroscopies and application to graphene. Phys Rev B. 2015;92:125147. https://doi.org/10. 1103/PhysRevB.92.125147.

Katz-Boon H, Rossouw CJ, Dwyer C, Etheridge J. Rapid measurement of nanoparticle thickness profiles. Ultramicroscopy. 2013;124:61-70. https://doi. org/10.1016/j.ultramic.2012.08.009.

Kim H, Zhang JY, Raghavan S, Stemmer S. Direct observation of Sr vacancies in $\mathrm{SrTiO}_{3}$ by quantitative scanning transmission electron microscopy. Phys Rev X. 2016;6:041063-7. https://doi.org/10.1103/PhysRevX.6.041063.

Kim Y-M, He J, Biegalski MD, et al. Probing oxygen vacancy concentration and homogeneity in solid-oxide fuel-cell cathode materials on the subunit-cell level. Nat Mater. 2012;11:1-7. https://doi.org/10.1038/nmat3393.

Kim Y-M, Pennycook SJ, Borisevich AY. Quantitative comparison of bright field and annular bright field imaging modes for characterization of oxygen octahedral tilts. Ultramicroscopy. 2017;181:1-7. https://doi.org/10.1016/j. ultramic.2017.04.020

Kimoto K, Asaka T, Yu X, et al. Local crystal structure analysis with several picometer precision using scanning transmission electron microscopy. Ultramicroscopy. 2010;110:778-82. https://doi.org/10.1016/j.ultramic.2009.11.014. 
Komsa H-P, Kotakoski J, Kurasch S, et al. Two-dimensional transition metal dichalcogenides under electron irradiation: defect production and doping. Phys Rev Lett. 2012;109:035503-5. https://doi.org/10.1103/PhysRevLett.109.035503.

Komsa H-P, Kurasch S, Lehtinen O, et al. From point to extended defects in twodimensional $\mathrm{MoS}_{2}$ : evolution of atomic structure under electron irradiation. Phys Rev B. 2013:88:035301-8. https://doi.org/10.1103/PhysRevB.88.035301.

Kovarik L, Stevens A, Liyu A, Browning ND. Implementing an accurate and rapid sparse sampling approach for low-dose atomic resolution STEM imaging. Appl Phys Lett. 2016;109:164102-6. https://doi.org/10.1063/1.4965720.

Krivanek OL, Lovejoy TC, Dellby N, et al. Vibrational spectroscopy in the electron microscope. Nature. 2014;514:209-12. https://doi.org/10.1038/nature13870.

Kurasch S, Kotakoski J, Lehtinen $\mathrm{O}$, et al. Atom-by-atom observation of grain boundary migration in graphene. Nano Lett. 2012;12:3168-73. https://doi. org/10.1021/nl301141g.

Lagos MJ, Trügler A, Hohenester U, Batson PE. Mapping vibrational surface and bulk modes in a single nanocube. Nature. 2017;543:529-32. https://doi.org/ 10.1038/nature21699

Lazić I, Bosch EGT, Lazar S. Phase contrast STEM for thin samples: integrated differential phase contrast. Ultramicroscopy. 2016;160:265-80. https:/doi.org/ 10.1016/j.ultramic.2015.10.011.

LeBeau JM, Findlay SD, Allen LJ, Stemmer S. Standardless atom counting in scanning transmission electron microscopy. Nano Lett. 2010;10:4405-8. https://doi.org/10.1021/nl102025s.

Lee J, Zhou W, Pennycook SJ, et al. Direct visualization of reversible dynamics in a $\mathrm{Si}_{6}$ cluster embedded in a graphene pore. Nat Commun. 2013;4:1650-7. https://doi.org/10.1038/ncomms2671.

Lehtinen $\mathrm{O}$, Vats N, Algara-Siller G, et al. Implantation and atomic-scale investigation of self-interstitials in graphene. Nano Lett. 2015;15:235-41. https://doi.org/10.1021/nl503453u.

Li C, Poplawsky J, Yan Y, Pennycook SJ. Understanding individual defects in CdTe thin-film solar cells via STEM: from atomic structure to electrical activity. Mater Sci Semicond Process. 2017;65:64-76. https://doi.org/10.1016/j.mssp. 2016.06.017.

Lin J, Cretu O, Zhou W, et al. Flexible metallic nanowires with self-adaptive contacts to semiconducting transition-metal dichalcogenide monolayers. Nat Nanotechnol. 2014;9:436-42. https://doi.org/10.1038/nnano.2014.81.

Lin J, Gomez L, de Weerd C, et al. Direct observation of band structure modifications in nanocrystals of $\mathrm{CsPbBr}_{3}$ perovskite. Nano Lett. 2016;16:7198202. https://doi.org/10.1021/acs.nanolett.6b03552.

Martinez GT, Rosenauer A, De Backer A, et al. Quantitative composition determination at the atomic level using model-based high-angle annular dark field scanning transmission electron microscopy. Ultramicroscopy. 2014; 137:12-9. https://doi.org/10.1016/j.ultramic.2013.11.001.

Mehdi BL, Qian J, Nasybulin E, et al (2015) Observation and quantification of nanoscale processes in Lithium batteries by operando electrochemical (S)TEM. Nano Lett 15:2168-2173 doi: https://doi.org/10.1021/acs.nanolett. $5 \mathrm{~b} 00175$

Meyer JC, Kotakoski J, Mangler C. Atomic structure from large-area, low-dose exposures of materials: a new route to circumvent radiation damage. Ultramicroscopy. 2014;145:13-21. https://doi.org/10.1016/j.ultramic.2013.11.010.

Miao J, Ercius P, Billinge $S$. Atomic electron tomography: 3D structures without crystals. Science. 2016; https://doi.org/10.1126/science.aaf2157.

Müller K, Krause FF, Béché A, et al. Atomic electric fields revealed by a quantum mechanical approach to electron picodiffraction. Nat Commun. 2014;5:5653. https://doi.org/10.1038/ncomms6653.

Ortalan V, Uzun A, Gates BC, Browning ND. Towards full-structure determination of bimetallic nanoparticles with an aberration-corrected electron microscope. Nat Nanotechnol. 2010;5:843-7. https://doi.org/10.1038/nnano.2010.234.

Oxley MP, Kapetanakis MD, Prange MP, et al. Simulation of probe positiondependent electron energy-loss fine structure. Microsc Anal. 2014;20:784-97. https://doi.org/10.1017/S1431927614000610.

Oxley MP, Lupini AR, Pennycook SJ. Ultra-high resolution electron microscopy. Rep Prog Phys. 2016;80:026101-65. https://doi.org/10.1088/ $1361-6633 / 80 / 2 / 026101$.

Pennycook SJ. Fulfilling Feynman's dream: "make the electron microscope 100 times better" —are we there yet? MRS Bull. 2015;40:71-8. https://doi.org/10. 1557/mrs.2014.307

Pennycook SJ. Imaging in STEM. In: Carter CB, Williams DB, editors. Transmission electron microscopy. 1st ed. Cham: Springer; 2016. p. 283-342.

Pennycook SJ, Boatner LA. Chemically sensitive structure-imaging with a scanning transmission electron microscope. Nature. 1988;336:565-7.
Pennycook SJ, Jesson DE. High-resolution incoherent imaging of crystals. Phys Rev Lett. 1990;64:938-41.

Pennycook SJ, Jesson DE. High-resolution Z-contrast imaging of crystals. Ultramicroscopy. 1991;37:14-38.

Pennycook SJ, Kalinin SV. Hasten high resolution. Nature. 2014;515:487-8. Pennycook SJ, Kalinin SV (2017) Single atom fabrication with beams and probes.

Pennycook SJ, Nellist PD, editors. Scanning transmission electron microscopy. New York, NY: Springer New York; 2011.

Pennycook TJ, Lupini AR, Yang $\mathrm{H}$, et al. Efficient phase contrast imaging in STEM using a pixelated detector. Part 1: experimental demonstration at atomic resolution. Ultramicroscopy. 2015;151:160-7. https://doi.org/10.1016/j.ultramic. 2014.09.013.

Pennycook TJ, Yang $H$, Jones $L$, et al. 3D elemental mapping with nanometer scale depth resolution via electron optical sectioning. Ultramicroscopy. 2017; 174:27-34. https://doi.org/10.1016/j.ultramic.2016.12.002

Prange MP, Oxley MP, Varela M, et al. Simulation of spatially resolved electron energy loss near-edge structure for scanning transmission electron microscopy. Phys Rev Lett. 2012;109:246101. https://doi.org/10.1103/ PhysRevLett.109.246101.

Rose H. Phase-contrast in scanning transmission electron microscopy. Optik. 1974;39:416-36.

Rutte RN, Jones L, Simson M, et al. Simultaneous atomic-resolution electron ptychography and Z-contrast imaging of light and heavy elements in complex nanostructures. Nat Commun. 2016;7:1-8. https:/doi.org/10.1038/ ncomms 12532

Salafranca J, Rincón J, Tornos J, et al. Competition between covalent bonding and charge transfer at complex-oxide interfaces. Phys Rev Lett. 2014;112: 196802.

Sang $X$, Oni AA, LeBeau JM. Atom column indexing: atomic resolution image analysis through a matrix representation. Microsc Anal. 2014;20:1764-71. https://doi.org/10.1017/S1431927614013506.

Shibata N, Findlay SD, Kohno Y, et al. Differential phase-contrast microscopy at atomic resolution. Nat Phys. 2012;8:611-5. https://doi.org/10.1038/ nphys 2337.

Shibata N, Seki T, Sanchez-Santolino G, et al. Electric field imaging of single atoms. Nat Commun. 2017;8:15631. https://doi.org/10.1038/ncomms15631.

Stevens A, Yang H, Carin L, et al. The potential for Bayesian compressive sensing to significantly reduce electron dose in high-resolution STEM images. Microscopy. 2014;63:41-51. https://doi.org/10.1093/jmicro/dft042.

Tang YL, Zhu YL, Hong ZJ, et al. 3D polarization texture of a symmetric 4-fold flux closure domain in strained ferroelectric $\mathrm{PbTiO}_{3}$ films. J Mater Res. 2016; 32:957-67. https://doi.org/10.1557/jmr.2016.259.

Tang YL, Zhu YL, Ma XL, et al. Observation of a periodic array of flux-closure quadrants in strained ferroelectric $\mathrm{PbTiO}_{3}$ films. Science. 2015;348:547-51. https://doi.org/10.1126/science.1258289.

Van Aert S, De Backer A, Martinez G, et al. Procedure to count atoms with trustworthy single-atom sensitivity. Phys Rev B. 2013;87:064107. https://doi. org/10.1103/PhysRevB.87.064107.

Varela M, Gazquez J, Pennycook SJ. STEM-EELS imaging of complex oxides and interfaces. MRS Bull. 2012;37:29-35. https://doi.org/10.1557/mrs.2011.330.

Varela M, Leon C, Santamaria J, Pennycook SJ. Scanning transmission electron microscopy of oxides. In: Tsymbal EY, Dagotto ERA, Eom C-B, Ramesh R, editors. Multifunctional oxide heterostructures. Oxford: Oxford University Press; 2017. p. 1-51.

Varela M, Oxley MP, Luo W, et al. Atomic-resolution imaging of oxidation states in manganites. Phys Rev B. 2009;79:085117. https://doi.org/10.1103/PhysRevB. 79.085117.

Voyles PM. Informatics and data science in materials microscopy. Curr Opin Solid State Mater Sci. 2017;21:141-58. https://doi.org/10.1016/j.cossms.2016.10.001.

Wang Y, Salzberger U, Sigle W, et al. Ultramicroscopy. Ultramicroscopy. 2016;168: 46-52. https://doi.org/10.1016/j.ultramic.2016.06.001.

Wang Z, Santhanagopalan D, Zhang W, et al. In situ STEM-EELS observation of nanoscale interfacial phenomena in all-solid-state batteries. Nano Lett. 2016; 16:3760-7. https://doi.org/10.1021/acs.nanolett.6b01119.

Wu B, Wu H, Wu J, et al. Giant piezoelectricity and high Curie temperature in nanostructured alkali niobate lead-free piezoceramics through phase coexistence. J Am Chem Soc. 2016;138:15459-64. https://doi.org/10. 1021/jacs.6b09024.

Yang Y, Chen C-C, Scott MC, et al. Deciphering chemical order/disorder and material properties at the single-atom level. Nature. 2017:542:75-9. https:// doi.org/10.1038/nature21042. 
Yang Z, Yin L, Lee J, et al. Direct observation of atomic dynamics and silicon doping at a topological defect in graphene. Angew Chem Int Edit. 2014;53: 8908-12. https://doi.org/10.1002/anie.201403382.

Yankovich AB, Berkels B, Dahmen W, et al. Picometre-precision analysis of scanning transmission electron microscopy images of platinum nanocatalysts. Nat Commun. 2014;5:1-7. https://doi.org/10.1038/ ncomms5155.

Yedra L, Eljarrat A, Arenal R, et al. EEL spectroscopic tomography towards a new dimension in nanomaterials analysis. Ultramicroscopy. 2012;122:12-8. https:// doi.org/10.1016/j.ultramic.2012.07.020.

Zhang JY, Hwang J, Isaac BJ, Stemmer S. Variable-angle high-angle annular darkfield imaging: application to three-dimensional dopant atom profiling. Sci Rep. 2015:1-10. https://doi.org/10.1038/srep12419.

Zheng T, Wu H, Yuan Y, et al. The structural origin of enhanced piezoelectric performance and stability in lead free ceramics. Energy Environ Sci. 2017;10: 528-37. https://doi.org/10.1039/C6EE03597C.

Zhou W, Lee J, Nanda J, et al. Atomically localized plasmon enhancement in monolayer graphene. Nat Nanotechnol. 2012;7:161-5. https://doi.org/10. 1038/NNANO.2011.252

Zhou W, Oxley MP, Lupini AR, et al. Single atom microscopy. Microsc Anal. 2012; 18:1342-54. https://doi.org/10.1017/S1431927612013335.

Zhou W, Pennycook SJ, Idrobo J-C. Localization of inelastic electron scattering in the low-loss energy regime. Ultramicroscopy. 2012;119:51-6. https://doi.org/ 10.1016/j.ultramic.2011.11.013

\section{Submit your manuscript to a SpringerOpen ${ }^{\circ}$ journal and benefit from:}

- Convenient online submission

- Rigorous peer review

- Open access: articles freely available online

- High visibility within the field

- Retaining the copyright to your article

Submit your next manuscript at $\gg$ springeropen.com 